\title{
Les premières années du Conservatoire et l'invention de la gestion
}

The first years of the Conservatoire and the invention of management

\section{François Vatin}

\section{(2) OpenEdition}

1 Journals

Édition électronique

URL : http://journals.openedition.org/artefact/3807

DOI : $10.4000 /$ artefact.3807

ISSN : 2606-9245

Éditeur :

Association Artefact. Techniques histoire et sciences humaines, Presses universitaires du Midi

\section{Édition imprimée}

Date de publication : 15 octobre 2019

Pagination : 31-51

ISBN : 978-2-8107-0650-1

ISSN : 2273-0753

\section{Référence électronique}

François Vatin, «Les premières années du Conservatoire et l'invention de la gestion », Artefact [En ligne], 10 | 2019, mis en ligne le 06 août 2020, consulté le 29 novembre 2020. URL : http:// journals.openedition.org/artefact/3807 ; DOI : https://doi.org/10.4000/artefact.3807

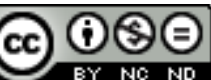

Artefact, Techniques, histoire et sciences humaines est mise à disposition selon les termes de la Licence Creative Commons Attribution - Pas d'Utilisation Commerciale - Pas de Modification 4.0 International. 


\section{Les premières années du Conservatoire et l'invention de la gestion}

\section{François Vatin}

\section{Résumé}

La gestion n'apparaît comme discipline académique en France que dans la seconde moitié du xIxe siècle. Pourtant, sous la Restauration, au Conservatoire des Arts et Métiers de Paris et dans les cours publics de Metz, on délivre, sous le nom d'« économie industrielle ", un enseignement que l'on peut comparer à la gestion. Les principaux auteurs de cette discipline sont Claude-Lucien Bergery, Gérard Joseph Christian, Charles Dupin et Jean-Baptiste Say. Cet article vise à exposer les idées centrales de l'économie industrielle des années 1820-1830, et à expliquer pourquoi cette discipline ne se développe pas dans les décennies suivantes du xixe siècle.

\section{Mots-clés}

Bergery (Claude-Lucien), Conservatoire des arts et métiers, Christian (Gérard Joseph), division du travail, Dupin (Charles), économie industrielle, enseignement professionnel, étalonnage, machinisme, Say (Jean-Baptiste)

99 François Vatin, « Les premières années du Conservatoire et l'invention de la gestion », Artefact, 10, 2019, p. 31-51. 


\section{The first years of the Conservatoire and the invention of management}

\section{Abstract}

Management appears as an academic discipline in France only in the second half of the nineteenth century. However, during the Restauration, in the Conservatoire des Arts et Métiers in Paris and in the public lectures in Metz, a teaching is delivered under the name of "industrial economics", that can be compared to management. The main authors of this discipline are Claude-Lucien Bergery, Gerard Joseph Christian, Charles Dupin and Jean-Baptiste Say. This article aims to expose the central ideas of the industrial economic of the years 1820-1830 and to explain why this discipline does not develop in the following decades of the $19^{\text {th }}$ century.

\section{Keywords}

Bergery (Claude-Lucien), Conservatoire des arts et métiers, Christian (Gérard Joseph), division of labour, Dupin (Charles), industrial economics, professional education, calibration, machinery, Say (Jean-Baptiste) 


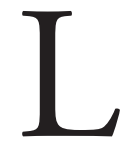

e terme de gestion est présent dès la première édition du Dictionnaire de l'Académie française en 1694 dans le sens d'administration. Cette définition ne bouge pas jusqu'à la huitième édition en 1934. Ce même dictionnaire définit le verbe "gérer " par "gouverner, conduire, administrer ", en précisant : "il n'est guère en usage que dans le Palais ». L'édition de 1762, donne aussi "gérer une tutelle ", celle de 1798 " gérer ses affaires ", et celle de 1835 " gérer un domaine, un établissement ». La notice reste ensuite constante jusqu'à la huitième édition du Dictionnaire en 1932. Lidée de gérer est donc passée progressivement du domaine public au domaine privé. Elle n'est que depuis peu de temps, associée au champ de l'entreprise. Quant à la gestion, elle n'est apparue que très récemment dans la cartographie académique française, puisque ce n'est qu'en 1976 qu'est ouverte une agrégation spécifique pour recruter les professeurs des universités ${ }^{1}$. Il n'y a pas lieu de s'en étonner, car le concept d'entreprise, dans le sens d'institution, est lui-même récent. Il ne s'agit pas d'un retard lexical, mais d'une question conceptuelle. Ronald Coase a montré en 1937 que, dans une économie où les agents ne seraient liés que par le marché, la notion d'entreprise ne pourrait avoir aucune place 2 . À sa suite, Oliver Williamson a insisté sur la différence entre les relations marchandes et les relations organisationnelles, qui reposent sur un principe de hiérarchie ${ }^{3}$. Cette question est corrélative de celle du salariat, que les juristes ont défini par la "subordination juridique et technique ", c'est-à-dire, par un type de liens contradictoires avec l'idéal libéral. Même si la gestion a ensuite abordé d'autres domaines de la vie de l'entreprise, elle prend sa source au début $\mathrm{du} \mathrm{Xx}^{\mathrm{e}}$ siècle dans le mouvement de l'organisation scientifique du travail, dont l'objectif affiché est de tirer le meilleur parti possible de la ressource humaine.

Ce préambule vise à mettre en lumière par contraste un moment mal connu de la pensée gestionnaire en France, à l'aube de la Révolution industrielle, sous la Restauration. Un petit groupe d'auteurs développe alors, sous l'expression d'économie industrielle, un discours qui préfigure la gestion. Ces principaux acteurs sont au Conservatoire des Arts et Métiers : Gérard Joseph Christian, son directeur, et deux de ses professeurs : Charles Dupin et Jean-Baptiste Say, auxquels il faut adjoindre Claude-Lucien Bergery, qui

\footnotetext{
1. Marco, 2006.

2. Coase, 1987.

3. Williamson, 1994.
} 
fonde à la même époque à Metz un enseignement pour ouvriers. Pourtant, ce champ intellectuel ne se développa pas dans les décennies suivantes et il fallut attendre le début du $\mathrm{Xx}^{\mathrm{e}}$ siècle pour que ces questions soient reposées dans le contexte du débat sur le Taylorisme. Je voudrais expliquer cette éclipse historique. On m'excusera de devoir dans ce bref article ne présenter que le cadre général et traiter comme un bloc ce groupe d'auteurs sur l'ensemble de la période de la Restauration, en négligeant délibérément les modalités de circulation des idées entre eux, les nuances qui les séparent et l'évolution de la question sur un temps qui reste bref. Pour la même raison, j'ai privilégié les citations pour faire connaître dans le texte les problématiques de ces auteurs.

\section{L'économie industrielle et l'instruction des industrieux}

Le mouvement en faveur d'un enseignement professionnel commence sous la Révolution. Le 15 septembre 1793, en pleine Terreur, une pétition du département de Paris sur l'enseignement professionnel, soutenue par les Jacobins, suscite une virulente opposition des Montagnards qui, comme Cambon, s'indignent qu'on voulût " faire croire qu'on ne peut bien faire un soulier qu'un compas à la main et dans une académie... $"^{4}$. La République n'avait besoin, ni de savants, ni de techniciens! Il fallut attendre Thermidor pour que les Républicains modérés qui prennent alors le pouvoir promeuvent l'enseignement technique. En 1799, Jean Chaptal recommande, pour résister à la concurrence industrielle anglaise, la création de quatre écoles consacrées aux arts chimiques. L'année suivante, il propose au Tribunat, la création de quatre chaires au Conservatoire : mécanique et hydraulique; art de la construction des machines et des outils; chimie appliquée aux arts; art du dessin et envisage la duplication de ces cours dans les grandes villes de province ${ }^{6}$. Ce projet, repris en 1819 sur la recommandation d'un comité composé d'Arago, Thénard et Clément-Desormes, aboutit à la création de trois chaires : mécanique appliquée aux arts pour Charles Dupin, chimie industrielle pour Nicolas Clément-Desormes et

\footnotetext{
4. Cité par Leif et Rustin, 1954, p. 108-109.

5. Chaptal, an VIII.

6. Chaptal, an IX., p. 52-55.
} 
économie industrielle pour Jean-Baptiste Say ${ }^{7}$. Ce succès tardif tient beaucoup à l'action de Dupin ${ }^{8}$. En 1824, celui-ci obtient l'autorisation de réorienter sa chaire pour en faire un " cours normal » destiné à former des enseignants susceptibles de distribuer dans tout le pays la bonne parole industrielle9. Dans son cours d'ouverture du 14 décembre 1825, il montre, métier par métier, l'usage technique que l'on peut faire des connaissances arithmétiques, géométriques et mécaniques ${ }^{10}$. Mais, source du progrès technique, la formation ouvrière est aussi pour lui le gage d'un progrès social et moral.

L'économie industrielle est pour ces auteurs la discipline qui fait le lien entre la dimension technique et la dimension morale et politique de l'industrie. Elle trouve sa place dans la classification naturelle des connaissances humaines élaborée par André-Marie Ampère en 1834, où elle constitue une branche " du troisième ordre » d'une vaste technologie divisée en deux branches : la technologie élémentaire et la technologie comparée ${ }^{11}$. Elle y forme, avec la physique industrielle, la technologie comparée. La technologie élémentaire se compose de la technographie (science des procédés) et de la cerdonistique industrielle (science du calcul appliquée à l'industrie $)^{12}$. Chez les auteurs qui nous intéressent ici, l'économie industrielle repose sur une philosophie de la production, développée par Jean-Baptiste Say dans son Traité d'économie politique en $1803^{13}$. Lointainement issue de la philosophie de Francis Bacon ${ }^{14}$, et, plus immédiatement, de la chimie de Lavoisier et de la Physiocratie, cette doctrine affirme que l'activité économique ne saurait créer de la matière. L'homme canalise à son profit des

7. «Ordonnance du roi portant établissement, au Conservatoire des arts et métiers, d'un enseignement public et gratuit pour l'application des sciences aux arts industriels ", cité dans Charmasson, Lelorrain et Ripa, 1987, p. 116-119.

8. Voir Dupin, 1839, p. 18-19.

9. Voir Dupin, 1825, tome 2, p. 245.

10. Dupin, 1826, p. 87 et sq.

11. Voir Ampère, 1834, p. 81-82. Ampère conçoit la classification des connaissances humaines comme analogue à la classification des espèces de Linné ( $\mathrm{p}$. XXXII et sq.) et distingue trois niveaux d'embranchement qui lui fournissent les nomenclatures des sciences du premier, deuxième et troisième ordres.

12. L'économie industrielle des auteurs que nous analysons ici recouvre donc l'économie et la cerdonistique industrielles d'Ampère.

13. Vatin, 2002.

14. On trouve chez Say (1840, p. 4) des références appuyées à Bacon; de même, Christian (1819, p. 33) met un paragraphe de Bacon en exergue de son ouvrage. 
forces naturelles, y compris celles de son propre corps, qui sont constantes, pour créer de l'utilité (" qualité de pouvoir servir»). "Production n'est point création ; c'est production d'utilité ${ }^{15}$.

Science pratique de l'organisation technique, économique et sociale de la production, l'économie industrielle organise, au-delà du cours de Say, l'ensemble des enseignements du Conservatoire. Elle est très présente chez Charles Dupin, mais aussi chez Gérard Joseph Christian, qui publie en 1819 ses Vues sur le système général des opérations industrielles, ou plan de technonomie ${ }^{16}$. Cette Technonomie se compose de trois branches : "l'économie domestique, qui a pour but de dépenser journellement moins que son revenu, sous quelque forme qu'il ait lieu "; "l'économie industrielle, dont le but est de produire le plus, avec le moins de dépense possible ; "l'économie politique, qui ne peut avoir pour but que l'aisance générale ${ }^{17}$. Il distingue au sein de l'économie industrielle, l'économie rurale, l'économie manufacturière et l'économie commerciale, mais centre son propos, dans la seconde partie de son ouvrage, sur la seule économie manufacturière ${ }^{18}$.

À Metz, Bergery construit une synthèse des apports de Christian, Say et Dupin $^{19}$. Il crée en 1825 un enseignement gratuit pour ouvriers dans un esprit similaire à celui du Conservatoire et, dans ce cadre, ouvre en 1828 un cours d'économie industrielle. Dès 1826, il présente cet enseignement comme la clé de voûte de son projet :

Mais au-dessus des théories particulières, se trouve une théorie générale qui les domine toutes, une théorie qui est comme l'âme de toutes les industries, puisque sans elle, les travaux, bien loin de conduire à la fortune, mènent d'autant plus vite à une ruine complète, qu'ils ont plus d'étendue. Cette théorie importante, indispensable pour tous les fabricants, pour tous les ouvriers, pour

15. Say, 1803, p. 27. Voir de même Christian, 1819, p. 40 ou Bergery, 1833, tome 2, p. 15-16.

16. La Technonomie a été considérée par Guillerme et Sebestik, 1968 comme un ouvrage fondateur de la technologie moderne.

17. Christian, 1819, p. 101. Cette typologie recoupe celle de Say qui avait composé son Traité d'économie politique en trois volets : production, consommation et répartition.

18. L'enseignement du Conservatoire vise exclusivement l'industrie manufacturière. Mais, à la même époque sont créées les premières écoles agricoles où l'on enseigne l'économie rurale : Simonin, 2006.

19. Sur Claude-Lucien Bergery, Vatin, 2007. 
tout le monde peut-être, c'est celle de l'économie industrielle. Je me propose de professer cette science l'année prochaine, afin de remplir une immense lacune du système d'éducation ${ }^{20}$.

Ce cours se compose d'un premier volume consacré à l'économie de l'ouvrier (enseignée en 1828-1829) et de deux volumes consacrés à l'économie du fabricant (enseignée entre 1829 et 1831) ${ }^{21}$. En 1831, Bergery annonce comme sous presse deux autres volumes respectivement consacrés à l'Économie du commerçant et à l'Économie du cultivateur, qui ne paraîtront pas ${ }^{22}$. Cette construction est inspirée de la série du Petit producteur français, publiée par Dupin en 1826-1827, qu'il apprécie particulièrement ${ }^{23}$.

Pour Bergery, l'économie industrielle est une discipline morale :

L'économie industrielle, telle que je l'enseignerai, présentera les règles à suivre et les moyens à employer par toutes les classes de producteurs, pour arriver aussi promptement qu'il est possible à l'aisance et au bonheur social. Ces règles et ces moyens concernent deux genres de faits : les uns entièrement matériels, les autres purement moraux. J'ai cru qu'il ne suffisait pas de vous enseigner les sciences ; j'ai cru que nous avions à soigner encore autre chose que votre instruction ; j'ai cru enfin que l'éducation morale de l'ouvrier appelait aussi toute notre sollicitude ${ }^{24}$.

Cette morale de l'intérêt bien compris s'impose à tous, ouvriers comme fabricants :

Deux sentiments impérieusement prescrits par une autorité supérieure à toutes les autres, honorent, consacrent et rendent durable le succès d'un établissement industriel : une sollicitude paternelle,

\footnotetext{
20. Bergery, 1826, p. XV-XVI.

21. Voir Bergery, 1833.

22. L'économie de l'ouvrier de Bergery relève pour une part de l'économie domestique de Christian, mais, pour Bergery, l'ouvrier est d'abord un producteur. Bergery a peut-être aussi enseigné l'économie agricole à l'École normale de Metz, car cette discipline fait partie du programme qu'il recommande en 1835 ("Système raisonné de l'enseignement primaire », dans Bergery, 1847).

23. Après un volume introductif, Le Petit producteur français décline : Le petit propriétaire français (l'exploitant agricole) ; Le petit fabricant français (le manufacturier), Le petit commerçant français, L'ouvrier français, auxquels Dupin ajouta un sixième volume : L'ouvrière française. Voir Vatin, 2009. 24. Bergery, 1833, tome 1, p. 7.
} 
active pour le sort présent et futur de l'ouvrier, et une inflexible bonne foi dans les relations de l'établissement ${ }^{25}$.

\section{Entre la technique et l'économie : la science des modes généraux de fabrication}

Parmi les sources de l'économie industrielle, il faut aussi citer la Chimie appliquée aux arts de Jean Chaptal qui a déjà pour ambition de fournir la trame d'une technologie générale :

Le véritable moyen d'éclairer les arts consiste bien moins à en décrire les procédés avec exactitude, qu'à en ramener toutes les opérations à des principes généraux. [...] Un traité de chimie appliquée aux arts est donc un ouvrage de principes, et je croirais avoir atteint le but que je me suis proposé, si chaque artiste trouvait dans cet écrit la cause de tous ses résultats et la règle fondamentale de sa conduite ${ }^{26}$.

Chaptal reste toutefois en amont de l'activité du manufacturier :

Le premier [le chimiste] propose, le second [le fabricant] juge et décide. Ce qui paraît être le mieux pour le chimiste peut bien ne pas l'être pour le fabricant, parce que le chimiste ne prend conseil que de la science, tandis que le manufacturier connait ce qui existe, compare la dépense avec le produit de l'amélioration, etc. ${ }^{27}$

Say s'inspire de Chaptal dans son discours d'ouverture du cours du 2 décembre 1820 : «La mécanique et la chimie vous montrent ce que vous pouvez faire ; l'économie industrielle vous montre ce qu'il vous convient de faire ${ }^{28}$ ». Mais, là où Chaptal devait s'en remettre à la seule intuition du fabricant, il introduit précisément l'économie industrielle pour « coiffer »

25. Ibid. Christian (1819, p. 47) consacrait de même le sixième et dernier chapitre de la seconde partie de son ouvrage aux " rapports moraux du fabricant avec les ouvriers et avec les consommateurs ". 26. Chaptal, 1807, tome 1, p. XIX.

27. Ibid., p. IX-X. Le schéma de Chaptal évoque à cet égard celui de Joseph Schumpeter (1999), qui distingue la figure de l'inventeur de celle de l'entrepreneur innovateur, créateur de " nouvelles combinaisons".

28. Say, 1848, p. 143. 
les savoirs particuliers à chaque $\operatorname{art}^{29}$. Bergery reprend cette distinction entre savoirs spécifiques et savoirs généraux :

Ne confondez pas les modes généraux de fabrication avec les procédés. Les premiers s'appliquent à toute espèce d'entreprise ; ils en constituent la marche générale et dépendent uniquement des principes de l'économie industrielle. Les seconds sont des modes particuliers ; ils ne conviennent qu'au genre de fabriques pour lesquels on les a imaginés; ils dépendent, à la fois, de l'économie industrielle et des sciences physiques ou mathématiques dont ils sont des applications ${ }^{30}$.

La science des modes généraux de fabrication correspond en somme à l'économie industrielle d'Ampère. Mais Ampère n'alimentait cette rubrique que par l'exemple rebattu de la division du travail. Christian et Bergery proposent un programme nettement plus riche.

Pour Christian, le travail industriel se présente selon deux modes :

L'un consiste dans une simple opération de la main, armée le plus ordinairement d'un outil qu'elle dirige, et dont l'homme doit suivre plus ou moins attentivement l'action et l'effet, c'est ce qu'on appelle métier; l'autre, dans un certain nombre d'opérations simultanées, exercées, de concert, par des agents divers, choisis avec intelligence et disposés avec habileté pour arriver à un but unique, c'est ce qu'on appelle fabrique, usine, ou manufacture ${ }^{31}$.

Autrement dit, dans le passage du métier à la fabrique, ce qui est en cause, ce n'est pas l'état spécifique d'un art, mais une évolution plus fondamentale, économique et sociale, soit le " machinisme ${ }^{32}$ » :

Il y a, en effet, sous le rapport économique, une telle corrélation entre les métiers et les manufactures ou fabriques proprement dites qu'on peut, dans une seule vue, confondre ceux mêmes qui, sous le rapport technique, semblent essentiellement séparés ${ }^{33}$.

29. Ibid., p. 150.

30. Bergery, 1833, tome 2, p. 81.

31. Ibid., p. 44.

32. Voir sur les ambiguïtés de cette expression, Vatin, 2012, chapitre 5.

33. Christian, 1819, p. 110. 
Pour Bergery, la distinction entre mode général et procédés spécifiques recouvre la division du travail entre fabricant et ouvriers :

C'est le fabricant qui pratique le mode général selon lequel il exploite son industrie, puisqu'il distribue, en conséquence, le travail à ses ouvriers. Ce sont les ouvriers qui mettent en œuvre le procédé que le maittre croit devoir adopter pour obtenir des produits d'une certaine qualité et d'un certain prix ${ }^{34}$.

Il distingue trois grands modes de fabrication : la division du travail, l'emploi de machines et l'étalonnage :

D'après cela, forger le fer sous de gros marteaux et le convertir en barres sous des cylindres cannelés sont deux procédés de l'art des forges ; mouler en sable ou mouler en terre sont deux procédés du fondeur ; teindre avant le tissage ou teindre après sont deux procédés du drapier. Mais n’employer que des hommes pour effectuer le travail est un mode général de fabrication et substituer des machines au plus grand nombre de ces hommes en est un autre; faire confectionner un objet entièrement par un seul ouvrier n'est pas le même mode général que de diviser l'exécution en autant d'ouvriers qu'il y a de parties distinctes; enfin on suit un mode général contraire à celui qui est appelé étalonnage, lorsqu'il n'y a pas d'uniformité dans les formes ni dans les dimensions des produits ${ }^{35}$.

Pour Christian, la question de l'uniformisation des produits ${ }^{36}$, relève d'abord de la consommation. Il souligne à la manière d'Henry Ford que la variabilité des goûts est un obstacle à l'industrialisation et dénonce la soumission des fabricants aux " caprices " des consommateurs " :

La nature et la distinction des produits, ainsi que le goût extrêmement variable de ceux qui s'en servent ou qui les consomment, portent un grand obstacle au progrès de plusieurs arts industriels; on voit, en outre, le mode de production en usage donner lieu aux caprices des consommateurs et les servir sans nécessité

34. Ibid., p. 81-82.

35. Bergery, 1833, tome 2, p. 82.

36. Le terme étalonnage ne figure pas chez Christian, mais chez Say (Cours, 1840, p. 129-131) dans un passage inspiré de Christian ; il est ensuite repris par Bergery. 
comme sans fruit. [...] Si nous supposons maintenant qu'il y ait de grandes fabriques séparées pour chacun de ces objets [de menuiserie] en particulier, il est certain qu'une douzaine de variétés pour les dimensions, une douzaine de variétés pour le luxe, suffiront amplement à tous les besoins comme à tous les goûts : chacun pourrait choisir dans ces limites de formes et de dimensions ce qui lui conviendrait. Or cette sorte d'uniformité tournerait évidemment au profit de l'usage, au surcroît de commodité, et permettrait d'introduire, dans ce genre d'industrie, un système d'exécution plus parfait et assurément plus économique. [...] Ceci est le principal objet de ce qu'on peut nommer l'" esthétique industrielle " $^{37}$.

On trouve des propos analogues chez Say ou Bergery ${ }^{38}$. Mais, en outre, Bergery développe une analyse organisationnelle de l'étalonnage, inspirée par son expérience d'officier d'artillerie ${ }^{39}$ :

La plupart des produits de l'industrie manufacturière sont composés de plusieurs pièces et ces pièces, un même ouvrier ne peut les faire toutes à la fois ; il est, du moins, obligé de les préparer séparément, et après en avoir terminé une, il prend les mesures qui doivent le diriger dans la confection de la suivante ; autrement les deux pièces ne pourraient s'ajuster ensemble ou concourir à la formation du même objet. Or ces mesurages qu'il faut faire pour chaque partie d'un produit et recommencer pour celles d'un autre produit tout pareil, causent une grande dépense de temps. C'est donc un moyen sûr d'opérer une notable diminution dans les frais que d'employer un mode de fabrication qui fasse éviter la répétition des mesurages. Ce mode existe : on l'appelle étalonnage, parce qu'il consiste dans l'emploi d'un modèle, ou patron, ou gabarit, ou étalon exécuté avec soin. L’ouvrier présente de temps en temps au modèle, la pièce dont il s'occupe, vérifie rapidement les formes et les dimensions, n’hésite pas un instant dans son travail et parvient,

37. Christian, 1819 , p. 53-54 et 146. On retrouve l'expression d'esthétique industrielle en 1847 chez Laboulaye, comme synonyme d'" ornement ». Elle désigne après la seconde-guerre mondiale le design.

38. Voir Say, 1840, p. 129-131 ; Bergery, 1833, tome 2, p. 37-40.

39. C'est chez les artilleurs que se développe dès le XviII ${ }^{\mathrm{e}}$ siècle, une réflexion sur la normalisation du matériel, d'abord dans le domaine des canons avec le système de Gribeauval, puis dans le domaine des armes portatives (voir Bret, 2002, p. 167-200). 
tout en abrégeant la fabrication, à donner aux produits plus de précision et de beautét ${ }^{40}$.

Par ailleurs, il souligne les liens étroits entre l'étalonnage et les deux autres modes généraux de fabrication :

L'étalonnage peut être employé seul, mais il peut aussi joindre ses avantages à ceux de la division du travail et de l'emploi des machines que les hommes font mouvoir ; souvent même, on ne saurait se dispenser de combiner les deux premiers modes, afin que les pièces exécutées par des ouvriers différents se conviennent et s'assemblent avec précision. Quant aux machines ouvrières qui sont mises en jeu par les animaux ou les moteurs inanimés, elles se trouvent organisées de manière à donner toujours les mêmes formes et les mêmes dimensions aux produits : elles rendent donc l'étalonnage superflu ou plutôt ce mode est inhérent à leur emploi et constitue un de leurs principaux avantages, comme la division du travail ${ }^{41}$.

Au début du XIX ${ }^{\mathrm{e}}$ siècle, la thématique de la division du travail, popularisée par Smith en 1776, est un lieu commun de la pensée économique et sociale. Mais les technologues auxquels nous nous intéressons envisagent la question d'une façon plus concrète que le philosophe anglais qui usait de cette expression comme synecdoque de la société marchande. Ils reformulent en des termes technologiquement plus convaincants ses trois célèbres arguments : l'augmentation de l'habileté individuelle, la réduction du temps perdu du fait des changements de tâches, l'invention de machines. Parce qu'ils pensent le machinisme et la captation des énergies non-humaines, la division du travail ne leur apparaît pas comme l'alpha et l'oméga de l'organisation industrielle. Dès 1803, Say avait en effet critiqué Smith sur ce point $^{42}$. Christian souligne de plus, comme, à la même époque, Andrew Ure et, bien plus tard, les sociologues de l'automation ${ }^{43}$, que le machinisme une fois développé contredit la division du travail :

Lorsqu'on en vient, dans beaucoup de travaux, à substituer des machines à la main-d'œuvre ou à employer des procédés ou des

40. Bergery, 1833, tome 2, p. 121-122.

41. Bergery, 1833, tome 2, p. 123-124.

42. Say, 1817, p. XLIX-L.

43. Voir Ure, 1836, p. 29-30 ; Naville, 1963. 
appareils chimiques mieux entendus, plus perfectionnés, cette grande subdivision disparaît et l'on réunit avec avantage dans les mêmes mains la conduite de plusieurs opérations ${ }^{44}$.

C'est donc une analyse poussée du machinisme que l'on trouve chez ces auteurs. Christian distingue trois stades dans son développement. Le premier est énergétique : "donner à la force physique de l'homme toute la puissance possible " et "faire usage des forces inanimées pour [la] remplacer "; le second est cinématique : «trace[r] des combinaisons de mouvements au moyen desquelles [la mécanique] exécute complètement des travaux qui exigeraient le concours de plusieurs mains exercées »; le troisième est celui de l'organisation industrielle proprement dite :

Trouver des combinaisons mécaniques au moyen desquelles une main-d'œuvre quelconque puisse exécuter, de la manière la plus parfaite et sans apprentissage préalable, un travail ou des opérations qui exigent un long apprentissage ; ou bien trouver des combinaisons par lesquelles on diminue la fatigue ou les incommodités du travail, ou par lesquelles on n'ait à craindre ni le défaut d'attention, ni la mauvaise volonté des ouvriers dans les opérations qu’on leur confie ${ }^{45}$.

\section{La question salariale}

Il s'agit maintenant de comprendre pourquoi une analyse aussi développée n'a pas pu déboucher sur un savoir institutionnalisé. C'est ici que nous retrouvons, derrière la question de la division du travail, celle du salariat.

Le débat sur la division du travail relève en fait de deux registres souvent confondus. Le premier, qu'on vient d'examiner, relève de l'organisation industrielle. En la matière, l'économie industrielle française de la Restauration ne fournit aucun progrès analytique significatif par rapport à Smith ${ }^{46}$. Mais cette question gestionnaire est parasitée par un autre débat,

\footnotetext{
44. Christian, 1819, p. 110.

45. Christian, 1819, p. 112-114.

46. À la même époque, Charles Babbage (1833, p. 117) complète le schéma de Smith par un " quatrième principe ", celui du découpage hiérarchique des tâches : si le travail n'est pas divisé selon le niveau de difficulté, l'entrepreneur devra payer les tâches simples au tarif requis pour les tâches complexes.
} 
moral et social, relatif au salariat. Ce débat est marqué en France par la publication en 1801 d'un texte caustique d'inspiration contre-révolutionnaire de Pierre-Édouard Lemontey, qui développe trois arguments contre la division du travail :

- son influence "sur les agents qu'elle emploie " : la déqualification ;

- son influence "sur les agents qu'elle réforme", le chômage ;

- son influence enfin «sur le corps de la nation ", c'est-à-dire la concentration capitaliste et la polarisation de la structuration sociale au détriment de la classe moyenne ${ }^{47}$.

Le texte de Lemontey a été repris par tous les penseurs sociaux français du XIX ${ }^{\mathrm{e}}$ siècle, de Say à Durkheim, en passant par Sismondi, Proudhon, Michelet et de nombreux autres. Dès 1803, Say le plagie : "C'est un triste témoignage à se rendre que de n'avoir jamais fait que la dix-huitième partie d'une épingle ${ }^{48}$ ". Ce plagiat suscite l'ironie de Lemontey lors de la réédition de son ouvrage en 1829 . En réponse, Say consacre aux thèses de Lemontey un chapitre entier de son Cours $^{49}$. S'il le critique, il reconnaît qu'il a "indiscutablement raison sur plusieurs points". Il souligne, d'abord, que celui-ci aurait confondu "l'influence de la séparation des occupations avec l'influence des machines ", lesquelles, le plus souvent, ne simplifieraient pas le travail, mais diminueraient le nombre d'hommes mobilisés. Il précise ensuite que la division du travail conduit à une distribution sélective des travailleurs en fonction de leurs compétences. Il note aussi que la vie sociale de l'homme ne se limite pas à sa vie professionnelle. Enfin, il souligne que, dans les campagnes où le travail est moins divisé, la main-d'œuvre ne témoigne pas d'une "supériorité morale ou intellectuelle marquée ". Mais surtout il oppose à Lemontey un argument techno-économique : "Du moment que l'homme n'a plus à faire que la fonction d'une cheville ou d'une manivelle, on le décharge de cette fonction toute mécanique et l'on en charge un moteur ${ }^{50} »$. Il admet, en revanche, comme légitime l'argument de dépendance à l'égard de l'entrepreneur qu'il avait fait sien dès 1803 :

47. Voir Lemontey, 2006, p. 384-420.

48. Say, 1803, p. 79. La phrase est de Lemontey. Pour une analyse du plagiat de Say, voir Frobert, 2001, p. 1735-1757.

49. Voir Say, 1840 , p. 84-85.

50. Cette idée figure déjà chez Christian, 1819, p. 24. 
C'en est un plus grave [d'inconvénient] de rendre chaque travailleur, en particulier trop dépendant de ses confrères et des entrepreneurs d'industrie. Comme dépendant de ses confrères, son existence est précaire. Un homme qui sait faire des sabots peut faire des sabots partout; mais un homme qui ne sait faire que des cadrans de montre, s'il est conduit par la fortune dans un pays où il n'y a pas une fabrique d'horlogerie montée en grand, ne pourra rien faire du tout. (...) Comme dépendant de l'entrepreneur d'industrie, l'ouvrier qui ne fait qu'une partie d'un produit a ce désavantage que le nombre des concurrents qui ont besoin de son travail est borné à celui des entrepreneurs ; tandis que s'il faisait un produit tout entier, il tirerait avantage de la concurrence des consommateurs ${ }^{51}$.

Bergery reprend cette discussion en suivant Say de près et, comme lui, retient l'argument de la dépendance. S'il affirme l'autorité du fabricant comme chef de l'atelier, il n'en considère pas moins que l'ouvrier doit rester " libre ", c'est-à-dire économiquement autonome. Il doit pouvoir quitter son employeur pour obtenir une meilleure place, ou, mieux, s'établir à son compte, ce qui est pour lui la destinée de l'ouvrier «bien né ». Ce primat de la doctrine libérale sur la pensée organisationnelle s'affirme dans un chapitre très suggestif intitulé : " organisation des travaux ${ }^{52}$ ». Bergery y traite de la " décomposition de la production " avant d'aborder la mesure des temps opératoires puis les formes de rémunération. On est ici apparemment pleinement chez Taylor :

La durée de chaque opération élémentaire doit être soigneusement appréciée. S'il en est qui demande plus de temps que les autres, un temps double, par exemple, on affecte deux ouvriers à chacune des premières, et l'on confie chacune des secondes à un seul homme. [...] De cette manière, il n’y a jamais encombrement à aucun établi et personne ne peut rester séparément un moment oisif : il faudrait pour qu'il y eût du temps de perdu que tous les ateliers s'entendissent, que dans tous la suspension et la reprise du travail se fissent au même instant. Un tel accord n'est guère possible sous les yeux des surveillants, et s'il n'avait pas lieu, la paresse, la nonchalance

51. Say, 1840, p. 84.

52. Voir Bergery, 1833, tome 3, p. 40-49. 
même d'un ouvrier serait constatée par le chômage qu'elle occasionnerait au suivant ${ }^{53}$.

Marqué, comme tous les ingénieurs de son temps, par les études de Coulomb sur la force des hommes ${ }^{54}$, Bergery est toutefois conscient de la limite de la mesure chronométrique du travail. Pour équilibrer les tâches, il faut trouver le bon couple de paramètres (vitesse et effort) qui, pour chaque travail, maximise le produit pour une fatigue donnée : "On peut, pour chaque genre d'ouvrage, faire un effort et agir avec une vitesse qui produisent le plus grand travail dans un temps donné ou qui consument le moins de temps pour un travail donné " 55 . Pour fixer les temps opératoires de chaque tâche, il faudrait donc disposer de cette analyse préalable : "Ainsi, lorsque le fabricant a trouvé le degré d'effort et le degré de vitesse qui produisent le plus d'ouvrage en un jour, par exemple, il peut être certain que la journée est le temps strictement nécessaire à la production de l'ouvrage $^{56}$ ». Malheureusement ces données sont difficiles à produire, puisqu'elles sont propres à chaque " genre d'ouvrages »; "l'observation seule peut les faire connaître ${ }^{57}$ ».

C'est pourquoi, Bergery recommande finalement le " travail à la pièce » : "Vous avez au reste un moyen très simple de vous dispenser de toute recherche et d'obtenir sûrement, sans la moindre surveillance, le plus grand travail dont chaque ouvrier est capable : c'est de payer à la pièce ${ }^{58} "$. Les ouvriers seront ainsi spontanément portés à chercher les paramètres optimaux de leur travail ${ }^{59}$. Ce mode salarial lui apparaît ainsi comme le

53. Bergery, 1833 , tome 3, p. 41. On trouve des considérations similaires sur l'équilibrage de la production chez Babbage (1833, chapitre XxII).

54. Voir Coulomb, 1821 ; Bergery s'était penché sur la fatigue animale dès 1826 (Bergery et Migout, 1836).

55. Bergery, 1833, p. 43. Bergery a implicitement admis ici une hypothèse fondatrice du mémoire de Coulomb : l'assimilation du temps de travail social à un quantum de fatigue (voir Vatin, 1993, chapitre 2). Maximiser le produit pour un temps donné consiste donc à maximiser le produit pour une fatigue donnée.

56. Bergery, 1833, tome 3, p. 44. L'analyse mécanique du travail inspirée de Coulomb que propose Bergery est ainsi plus développée que celle de Taylor, qui ne dispose d'aucune mesure de la fatigue. 57. Bergery, 1833, tome 3, p. 45.

58. Bergery, 1833, tome 3, p. 46.

59. Lidée selon laquelle la norme marchande conduit les ouvriers à dégager eux-mêmes les paramètres optimaux de leur travail est au cœur de la démarche de Coulomb (voir sur ce point Vatin, 1993). 
moyen idéal pour compenser la faiblesse de l'organisation. Charles Dupin le recommandait aussi à son "petit fabricant français » :

M. Lerond mit [les ouvriers] à leurs pièces, en fixant le prix de la tâche sur le taux moyen du travail. Cela leur déplut, d'abord, parce que c'était nouveau. [...] D'abord, les ouvriers ne travaillèrent pas beaucoup plus qu'à la journée ; mais bientôt les occasions se présentèrent en foule pour leur faire désirer quelque argent. [...] Par ces stimulants, les ouvriers de M. Lerond acquirent bientôt toute l'activité désirable et finirent par gagner une fois autant que les journaliers du canton ${ }^{60}$.

Jean-Baptiste Say écrivait aussi :

Toutes les fois que la tâche est appréciable, on préfere les payer ainsi, parce que, pourvu que l'ouvrage soit bien exécuté (ce qu'on vérifie avec soin), on est assuré de ne pas payer plus de travail qu'il n'y en a eu de fait. Il est même indispensable de payer ainsi les travaux que l'ouvrier exécute chez lui, loin des yeux du maître et du contremaître $^{61}$.

Mais, dans les conditions ordinaires de surveillance du travail, Say n'accordait toutefois pas grande importance au mode de rémunération :

Mais comme l'expérience fait connaître bien vite à l'entrepreneur la quantité d'ouvrage qu'un ouvrier peut exécuter en un jour, il sait quel salaire journalier chacun de ses ouvriers peut gagner à la tâche ; et c'est sur le montant de cette journée que roule le débat qui s'élève pour le prix entre le maître et l'ouvrier ${ }^{62}$.

Or, c'est précisément cette hypothèse que Taylor a dénoncée ${ }^{63}$. Si les patrons sont portés à payer les ouvriers à la pièce, c'est parce qu'ils ne connaissent pas les conditions réelles du travail. Si les ouvriers augmentent leur rendement, les patrons baissent le prix des pièces. Pour se protéger de ce risque, les ouvriers pratiquent donc le freinage. Pour contrer ce mécanisme pervers, Taylor recommande donc l'analyse systématique du travail, c'est-à-dire ce à quoi Bergery avait renoncé du fait de sa confiance dans le

60. Dupin, 1827-1828, tome 3, p. 31.

61. Say, 1840 , p. 332

62. Say, 1840, p. 322.

63. Taylor, 1990. 
caractère stimulant du paiement à la pièce. Ainsi, les analyses de Say et de Bergery débouchent précisément sur le point de départ de Taylor, mais sur des modes différents. Say se situe dans le cadre d'un taylorisme abouti, où la question de la mesure du travail ne se pose plus. Bergery est en revanche convaincu de la faible connaissance qu'a le maître des conditions réelles du travail de l'ouvrier. Mais cette méconnaissance ne le choque pas, car elle correspond à sa conception de l'autonomie économique et technique de l'ouvrier. La résolution par le marché des conditions de l'échange travail/ salaire n'est pas pour lui un pis-aller, mais bien la solution idéale, économiquement comme moralement, si tant est que l'on puisse opposer ces deux niveaux chez cet auteur.

\section{Conclusion}

La question salariale permet de comprendre pourquoi ce moment de la pensée industrielle fut sans lendemain direct. Dans la France de la Restauration, la grande entreprise industrielle était rare. La gestion des hommes restait déconcentrée, passant par les mécanismes complexes du marchandage. On déléguait au mécanisme marchand la fonction de répartition du travail et de stimulation de la main-d'œuvre. L'enseignement de l'économie industrielle ne s'est donc pas développé au Conservatoire. Christian meurt en 1831. À la mort de Say en 1832, Bergery, qui avait été sollicité pour lui succéder, préféra rester à Metz. On élit sur cette chaire Adolphe Blanqui, qui développe un enseignement d'économie politique, laquelle n'était plus proscrite après 1830. En 1839, Dupin fait rebaptiser sa chaire Géométrie appliquée aux arts et statistique. C'est désormais l'économie politique, envisagée sous un registre protectionniste, qui mobilise également son attention. À la mort de Blanqui en 1854, c'est une chaire d'Administration et de statistique industrielle qui est confiée à Jules Burat. La thématique de l'organisation industrielle disparaît au profit de celle des " affaires " abordée dans une démarche de protection des intérêts économiques de la nation. Ce n'est que dans les années 1870 que commence à réapparaître en France une réflexion gestionnaire sur la conduite des hommes. Mais la thématique ne s'impose que quarante ans plus tard, lorsqu'eurent lieu les premières tentatives d'introduction en France de la " méthode Taylor». Le Conservatoire est alors à nouveau un lieu central du débat. Mais personne ne cherche à renouer avec les réflexions des premières années de l'institution. 


\section{Bibliographie}

AMPÈre André-Marie, Essai sur la philosophie des sciences ou exposition analytique d'une classification naturelle de toutes les connaissances humaines, Bachelier, Paris, 1834.

Babbage Charles, Traité sur l'économie des machines et des manufactures (1831), traduit de l'anglais sur la troisième édition par Édouard Biot, Bruxelles, 1833.

Bergery Claude-Lucien, Géométrie des courbes appliquées à l'industrie, Lamort, Metz, 1826.

Bergery Claude-Lucien, Économie industrielle ou science de l'industrie, 3 vol., $2^{e}$ éd. revue et augmentée, Thiel, Metz, 1833.

Bergery Claude-Lucien et Migout, Jean-Claude, Essai sur la théorie des affûts des voitures d'artillerie, Levrault, Paris et Strasbourg, 1836.

Bergery Claude-Lucien, Cosmographie des écoles primaires, $3^{\mathrm{e}}$ éd. revue et augmentée, Thiel, Metz, 1847.

BRET Patrice, L'État, l'armée et la science. L'invention de la recherche publique en France (1763-1830), Presses universitaires de Rennes, Rennes, 2002.

Chaptal, Jean, Essai sur le perfectionnement des arts chimiques en France, Paris, an VIII.

Chaptal Jean, Rapport et projet de loi sur l'instruction publique, Paris, brumaire an IX.

Chaptal Jean, Chimie appliquée aux arts, Déterville, Paris, 1807, 4 vol.

Charmasson Thérèse, Lelorrain Anne-Marie et Ripa Yannick, L'Enseignement technique de la Révolution à nos jours. Textes officiels avec introduction, notes et annexes, tome I, « De la Révolution à 1926 », Economica, Paris, 1987.

Christian Gérard Joseph, Vues sur le système général des opérations industrielles ou plan de technonomie, Huzard et Courcier, Paris, 1819.

CoAse, Ronald "La nature de la firme " (1937), Revue française d'économie, vol. 2-1, 1987, p. 133-163.

Соuцомв Charles-Augustin, "Résultats de plusieurs expériences destinées à déterminer la quantité d'action que les hommes peuvent produire par leur travail journalier " (1799) réédité dans Coulomb Charles-Augustin, Théorie des machines simples, Bachelier, Paris, 1821.

Dictionnaire de l'Académie française. Huit éditions (1694-1932).

Dupin Charles, Discours et leçons sur l'industrie, le commerce, la marine et sur les sciences appliquées aux arts, Bachelier, Paris, 1825.

Dupin Charles, Tableau des arts et métiers et des beaux-arts, Bachelier, Paris, 1826.

Dupin Charles Le Petit producteur français, Bachelier, Paris, 1827-1828, 6 vol. 
Dupin Charles, Historique de l'enseignement industriel et de son influence sur le sort du peuple de 1819 à 1839, Discours à la séance d'ouverture du 15 décembre 1839, Paris, 1839.

Frobert Ludovic « Pierre-Édouard Lemontey et la critique de la division du travail ", Économies et Sociétés « Histoire de la pensée économique », novembre-décembre 2001, p. 1735-1757.

Guillerme Jacques et Sebestik Jan, "Les commencements de la technologie », Thalès, tome XII, 1968, p. 1-72.

Laboulaye Charles (dir.), Encyclopédie technologique. Dictionnaire des arts et manufactures, Mathias, Paris, 1847.

LeIF Joseph et Rustin Georges, Histoire des institutions scolaires, Delagrave, Paris, 1954.

Lemontey Pierre-Édouard, "Influence morale de la division du travail, considérée sous le rapport de la conservation du gouvernement et de la stabilité des institutions sociales » (1801), réédition commentée par Vatin François, Revue du Mauss, n 27, 2006-1, p. 384-420.

Marco Luc, "L'agrégation de sciences de gestion (1976-2005) ", Revue d'histoire des sciences humaines, $\mathrm{n}^{\circ} 14,2006, \mathrm{p} .173-198$.

Naville Pierre, Vers l'automatisme social ?, Gallimard, Paris, 1963.

SAY Jean-Baptiste, Traité d'économie politique, Déterville, Paris, 1803.

SAY Jean-Baptiste, Traité d'économie politique, $3^{\mathrm{e}}$ éd. Déterville, Paris, 1817.

SAY Jean-Baptiste, Cours complet d'économie politique pratique (1828-1829), $2^{\text {nde }}$ éd., Hauman, Bruxelles, 1840.

SAY Jean-Baptiste, "Discours d'ouverture du cours d'économie industrielle » (décembre 1820) dans SAY Jean-Baptiste, Euvres, tome IV, Guillaumin, Paris, 1848, p. 133-147.

Schumpeter Joseph, Théorie de l'évolution économique (1911), Dalloz, Paris, 1999.

Simonin Jean-Pascal (dir.), Jean-Edmond Briaune, cultivateur, agronome, économiste, Presses universitaires d'Angers, Angers, 2006.

Sмiтh Adam, Essai sur la nature et les causes de la richesse des nations (1776), Garnier-Flammarion, Paris, 1991.

TAYlOR Frederick, "La direction des ateliers " (1903), repris dans VATIN François (éd.), Organisation du travail et économie des entreprises, Éditions d'organisation, Paris, 1990.

URE Andrew, Philosophie des manufactures ou économie industrielle de la fabrication du coton, de la laine, du lin et de la soie (1835), Hauman, Bruxelles, 1836.

VATIN François, Le travail, économie et physique, 1780-1830, Presses universitaires de France, Paris, 1993. 
Vatin François, "Pensée industrielle et théorie de la production chez JeanBaptiste Say ", dans Potier Jean-Pierre et Tiran André (éd.), Jean-Baptiste Say. Nouveaux regards sur son œeuvre, Economica, Paris, 2002, p. 605-628.

VATIN François, "Les aventures du bon Monsieur Lerond, le petit fabricant français ", dans Christen Carole et Vatin François (dir.), Charles Dupin (17841873), savant, économiste, pédagogue et parlementaire du Premier au Second Empire, Presses universitaires de Rennes, Rennes, 2009, p. 207-221.

Vatin François, Calcul et morale économiques dans le premier XIX siècle : L'économie industrielle de Claude-Lucien Bergery, L'Harmattan, Paris, 2007.

Vatin François, L'espérance-monde. Essais sur l'idée de progrès à l'heure de la mondialisation, Albin Michel, Paris, 2012.

Williamson Oliver, Les institutions de l'économie (1985), Inter-édition, Paris, 1994.

\section{L'auteur}

François Vatin est professeur de sociologie à l'université de Paris-Nanterre où il dirige le master de sciences économiques et sociales. Il est spécialiste d'histoire et de sociologie du travail, de l'économie et de la technique. Dans cette optique, il s'est particulièrement intéressé à la pensée des ingénieurs du xix siècle qui abordent d'un même mouvement les choses et les hommes dans leur volonté de concevoir une société plus rationnelle, c'est-à-dire tout à la fois plus efficace et plus juste. Il a publié de nombreux ouvrages dont parmi les derniers : L'espérancemonde. Essai sur l'idée de progrès à l'heure de la mondialisation (Albin Michel, 2012) et avec Gwenaële Rot, Au fil du flux. Le travail de surveillance-contrôle dans les industries chimiques et nucléaires (Presses des Mines, 2017). Il publiera prochainement avec Gwenaële Rot : Reynold Arnould. La poétique de l'industrie (Presses universitaires de Nanterre, 2019). 